\title{
Enhancing the Map Usage for Indoor Location-Aware Systems
}

\author{
Hui Wang ${ }^{1,2}$, Henning Lenz ${ }^{1}$, Andrei Szabo ${ }^{1}$, Joachim Bamberger ${ }^{1}$, \\ and Uwe D. Hanebeck ${ }^{2}$ \\ ${ }^{1}$ Siemens AG, Corporate Technology, Information and Communications, CT IC4, \\ Otto-Hahn-Ring 6, 81739, Munich, Germany \\ ${ }^{2}$ Intelligent Sensor-Actuator-Systems Laboratory, Institute of Computer Science and \\ Engineering, Universität Karlsruhe (TH), Germany \\ ${ }^{1}$ \{Hui.Wang.ext, Henning.Lenz, Andrei.Szabo, Joachim.Bamberger\}@ siemens.com \\ ${ }^{2}$ Uwe.Hanebeck@ieee.org
}

\begin{abstract}
Location-aware systems are receiving more and more interest in both academia and industry due to their promising prospective in a broad category of so-called Location-Based-Services (LBS). The map interface plays a crucial role in the location-aware systems, especially for indoor scenarios. This paper addresses the usage of map information in a Wireless LAN (WLAN)-based indoor navigation system. We describe the benefit of using map information in multiple algorithms of the system, including radio-map generation, tracking, semantic positioning and navigation. Then we discuss how to represent or model the indoor map to fulfill the requirements of intelligent algorithms. We believe that a vector-based multi-layer representation is the best choice for indoor location-aware system.
\end{abstract}

Keyword: Location-Aware Systems, WLAN Positioning, Map Representation

\section{Introduction}

Location-aware systems are receiving more and more interest in both academia and industry due to their promising prospective in a broad category of so-called locationbased services (LBS)[1-4], such as people and asset tracking, security applications, navigation and location-based multimedia services. Generally, current location-aware systems can be separated into three groups: satellite based systems, sensor based systems, and local network-based systems. Satellite systems, such as the well-known GPS or Galileo systems, focus on outdoor positioning using satellite signals. However, these systems suffer from the attenuation, reflection, and refraction of buildings and walls when used indoors. To solve this problem, two other kinds of systems use various sensors (e.g. infrared sensor, ultrasonic sensor or inertial sensor) and a local communication network interface (e.g. WLAN, Bluetooth, etc.) to collect indoor location information $[5,6]$.

In all location-aware systems, the map interface plays an important role, especially for indoor scenarios. This paper addresses the map information for indoor location- 
aware systems. We discuss two problems: 1) How indoor positioning systems can benefit maximally from the map information; 2) How to better model or represent the building maps in order to facilitate the whole positioning system.

In the GPS system, map matching methods are often used to evaluate the trajectories and project them onto streets $[7,8]$. In indoor systems, researchers also proposed dozens of map-assisted algorithms. For example, to generate the radio map, [6][9] used multi-wall and dominant path models and [10] used parametric raytracing. They all need to know the room and the wall information. For tracking algorithms, [11, 12] both use the map to delete impossible movements. Besides, [13, 14] used the Voronoi map to further restrict a possible trajectory. Other algorithms including [15-17] use maps to assist the sensor fusion.

The indoor positioning system in this paper is based on the emerging WLAN positioning techniques. Its basic structure consists of four layers, including the interface layer, the localization layer, the tracking and fusion layer, and the application layer, as shown in Fig. 1. In contrast to other systems, which typically only use the map in the application layer, we integrate map information into all layers of the system. In the interface layer, map information is used to generate a more accurate radio map. In the localization layer, we are able to estimate not only the user's geometrical location but also semantic location by using the map, e.g., the located floor and room. We also integrate the map information in the tracking level using particle filter, which can reduce the estimation variance by deleting some unrealistic cases like crossing the walls. Finally, map information is used in the application layer to support intelligent room detection, routing or other location/context aware services.

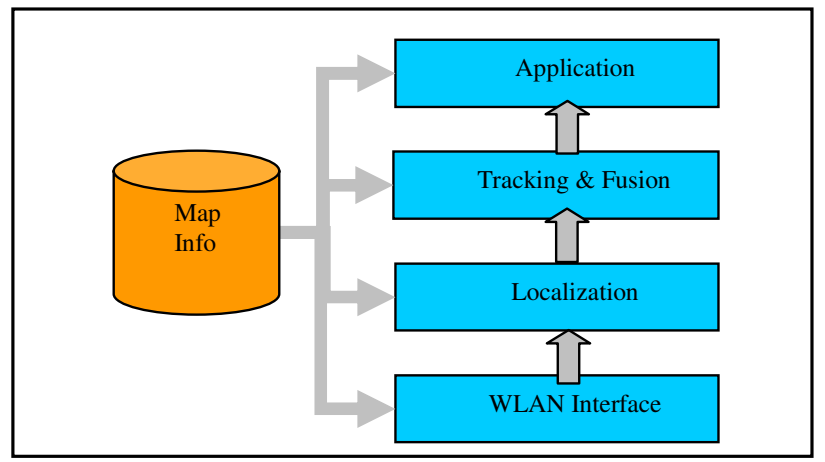

Fig. 1. Structure of a WLAN-based Location-aware System.

To facilitate the above mentioned map-related algorithm, the map information has to be well organized. To the best of our knowledge, there is still no standard for modeling and representing indoor map information. AutoCAD provides his own file format DWF (Design Web Format) for representing map information [18]. But since DWF aims at the general product design with a lot of redundant information, it is not suitable for localization systems. Other attempts from industry include SLoP (Spatial 
Location Protocol) by the Internet Engineer Task Force (IETF) [19]. It is a purely descriptive model, aiming at unambiguously expressing location information in an interoperable form in the Internet. Some researchers, such as [20-22], presented symbolic or semi-symbolic models to represent the location, which is similar to the model used in this paper.

In this paper, we use a vector-based multi-layer model to represent the indoor digital map. This model organizes the map based on three different aspects. The first is called geometric level, which includes points, lines or polygons. The second is symbolic level, including walls, doors, rooms or floors. The last is semantic level, including the semantic information, such as the name of employee inside the room, the room number, etc. In the symbolic level, the elements are organized using a hybrid tree and graph structure according to their geospatial relationships. The advantage of using this map representation is as follows. The wall or room information required by the localization and tracking algorithm can be easily extracted. In addition, the semantic information can fastly be outputted. Furthermore, the routing algorithm can be easily implemented by the hybrid tree and graph structure in symbolic level. Also, this model can be easily built by analyzing raster or CAD formatted files. Last but not the least, this model also facilitates the information exchange with web service, i.e. the model can also be easily represented by XML or SVG format.

The remainder of the paper is organized as follows. In Section 2, we introduce briefly the principle of WALN based localization system. Subsequently the advantages of using map information for assisting the whole system are discussed in detail. In Section 3, we describe the map representation used in our system and its advantages. Finally, Section 4 concludes the paper.

\section{Information Extraction from Maps for WLAN Localization}

\subsection{Principle of WLAN Localization Systems}

As described in Section 1, the WLAN localization systems can be separated into four layers: interface layer, localization layer, tracking \& fusion layer and application layer. In the following, we briefly describe the principle and the functionality of each layer. More details can be found in the literature [5, 6, 23, 24, 25].

- Interface Layer. In the interface layer, the system measures the WLAN signals and extracts the location-related information. The received signal strength (RSS) is typically used to infer the user's location because it is much easier to be obtained than the other information such as time of arrival (TOA), time difference of arrival (TDOA) or angle of arrival (AOA). The value of RSS is related to the distance between the access point (AP) and the user's mobile terminal. The longer the distance, the lower RSS will be measured. The 
distribution of RSS at numerous reference points can be measured or calculated before the system runs and is usually stored in a database named radio map.

- Localization Layer. In this layer, the mobile terminal compares the measurements with the RSS distributions in radio map. The reference point that has the most similar RSS distribution to the online measurement is finally chosen as the estimated position. This is the so called nearest neighbor method [5] [6]. Since the wireless channel is noisy, the accuracy of location estimation is limited. The output of this layer is usually a 2-D or 3-D local geometric coordinate. But with the help of map information, symbolic or semantic information (e.g. room number) is also obtainable.

- Tracking and Fusion Layer. If the mobile terminal is moving, the filtering algorithms can be used to smooth the moving trajectory. In addition, other information sources, such as extra sensors, building map and other locationrelated information, can also be fused with the assistance of some sophisticated but flexible filtering techniques like particle filters.

- Application Layer. The application layer interacts with the users directly. It applies the location information to support various location-based applications or services, such as asset monitoring, guidance, routing, map-services, locationdependant multimedia services or games, and etc. The way of operating the location information varies depending on different applications. But the basic operation is either to inform the user of location-related semantic information or to guide users to their target places.

\subsection{Map-Assisted Radio Map Generation}

Theoretically, the received signal power $P_{r}$ follows the radial propagation model, as described in Equation (1),

$$
P_{r}[d B m]=P_{r}\left(d_{0}\right)[d B m]-10 n \log \left(\frac{d}{d_{0}}\right)
$$

where $d$ is the distance between the mobile terminal and access point. $d_{0}$ is the reference distance, normally equal to 1 meter. $n$ is the attenuation parameter, which is equal to $n=2$ in free space and $n=1.5 \sim 6$ in indoor scenarios.

However, due to the complexity of indoor environments, the radial model is unrealistic for real radio propagation. Since the walls are the major obstacles attenuating the signal, a multi-wall model [26] as in Equation (2) can better model the radio propagation, where $W A F$ means the wall attenuation factor, which is the loss of signal power after crossing a wall.

$$
P_{r}[\mathrm{dBm}]=P_{r}\left(d_{0}\right)[\mathrm{dBm}]-10 n \log \left(\frac{d}{d_{0}}\right)-\left\langle\begin{array}{cc}
\sum W A F & l<k \\
C & l \geq k
\end{array}\right.
$$

Although the multi-wall model takes the environments into account, it only uses the direct ray between transmitter and receiver. This will lead to an underestimation in many cases. For instance, in Figure 2, the transmitter emits many possible rays to the 
receiver. The direct path penetrates three walls but there is also another indirect path penetrating only one wall. Since the signal on the direct path is attenuated a lot when crossing the walls, the signal on the indirect path contributes to most of the received power. So if we only consider the direct path, the predicted signal power at the receiver will be much lower than the real measurement. The dominant path model $[6$, 9] aims to find the signal propagation path, which dominates the received power. So the idea of the dominant path model is to find the path which has the smallest power loss. The calculation of the dominant path can be found in [6], which is similar to finding the shortest path between AP and mobile terminal.

Figure 3 and Table 1 show the generated radio maps and the positioning results using different channel models [6]. It is obvious that these site-specific models perform much better. For employing the multi-wall model or the dominant path model, the site-specific information, such as the position of the walls and rooms, should be extracted from the map. In addition, the map model should be well organized to facilitate the extraction of wall and room information.

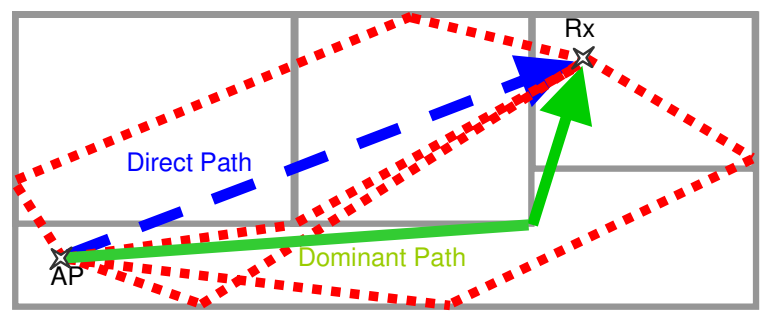

Fig. 2. Illustration of the Dominant Path Model.
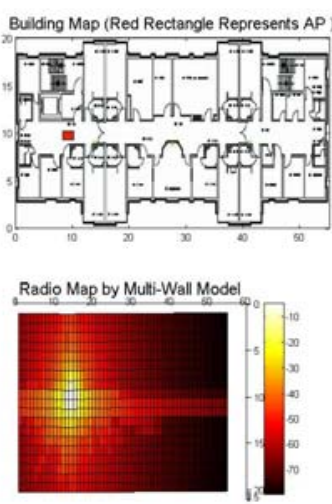

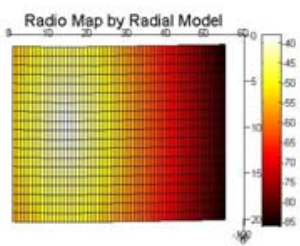

Radio Map by Dominant Path Model

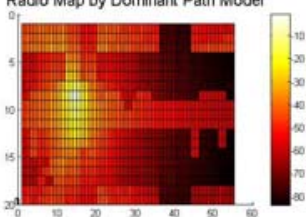

Fig. 3. Example of Radio Maps Using Different Channel Models. 
Table 1. Mean of Location Errors in a Testbed Using Different Channel Models.

\begin{tabular}{ll}
\hline Channel Models & Mean $(\mathrm{m})$ \\
\hline Linear Model & 7.11 \\
Mulit-Wall Model & 6.73 \\
Dominant Path Model & 5.70 \\
\hline
\end{tabular}

\subsection{Map-Assisted Tracking and Fusion}

Map information also improves the tracking and fusion process. The key idea is that the user cannot pass the walls while moving. In [11], we proposed a map-aided particle filter, which can incorporate the map information efficiently. The basic idea of this particle filter is as follows:

1. Use an amount of particles to represent the probabilistic distribution of user's position.

2. When the filter predicts the user's next step for each particle, the particles which crossed a wall are deleted.

Figure 4 illustrates the filtering process and Table 2 gives the tracking result with and without using map information in one of our testbeds.
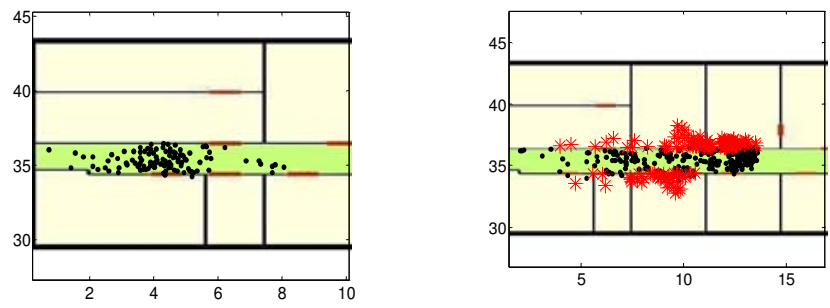

Fig. 4. Example of Two Succeeding Steps in Map-Aided Particle Filters. The red stars in right figure represent the deleted particles because they crossed a wall.

Table 2. Mean and Variance of Location Errors With and Without Using Map Information in a Testbed.

\begin{tabular}{lcc}
\hline & Mean $(\mathrm{m})$ & Variance $(\mathrm{m})$ \\
\hline Particle Filter without Map & 5.57 & 3.9 \\
Particle Filter with Map & 4.5 & 3.5 \\
\hline
\end{tabular}




\subsection{Map's Contribution to Semantic Localization and Navigation}

With the help of the map, location-based applications and services can automatically transform the geometric coordinate to high level semantic information. The topologic structure like a room connection matrix as depicted in Table 3 can facilitate the routing algorithm by simply using a Dijkstra or A* algorithm [6].

Table 3. Example of Room Connection Matrix.

\begin{tabular}{llll}
\hline & Room 1 & Room 2 & Corridor 1 \\
\hline Room 1 & - & No Connection & Door 1 \\
Room 2 & No Connection & - & Door 2 \\
Corridor 1 & Door 1 & Door 2 & - \\
\hline
\end{tabular}

\section{A Vector-Based Multi-Level Map Representation}

For achieving the benefits described in Section 2, the representation of map information should satisfy the following requirements: 1) Site-specific information, such as wall, room and floor information should be easy to extract. 2) The topologic structure should be easy to extract in order to assist intelligent routing. 3) The map representation should be easy to generate.

Typically, map information is organized in vector, raster, or a combined format. But to the best of our knowledge, there is still no standard map format for indoor location-aware systems. Most of the current systems simply plot the estimated position on the JPEG or BMP formatted building map. For this kind of map interface, the semantic information of indoor environment, e.g. room number, is actually identified by the user instead of location-aware systems. It makes advanced semantic based operation impossible, and thus does not fulfil our requirements.

In the paper, we use a vector representation of the indoor digital map. This model organizes the map based on three different aspects. The first is called geometric level, which includes points, lines, or polygons. The second is the symbolic level, including walls, doors, rooms, or floors. The last is the semantic level, including the semantic information, such as the room number, the access right, etc. This structure has the following characteristics.

1. Symbols are the core entities in our model. We define a symbol as having both geometric and semantic properties. For example, a symbol rooml has several geometric properties such as the position of the centre or the shape, and it has semantic properties such as the room number or the owner's name.

2. The symbols are organized hierarchically. In the example in Figure 5, the symbols are organized by a hybrid tree-graph structure according to their spatial relationship. In this structure, Floor is superset of room, elevator, or corridor. Room is a superset of door, wall, or other symbols inside a room.

3. Spatially, high level symbols include the low level symbols. In Figure 1, the symbol Elevator at Floor 1, Room1, Room2, and Corridorl are spatially inside the symbol Floor. 
4. The symbols in the same level are connected in a graph structure according to their spatial connection relationship. Like in Figure 5, Room1, and Room2 are connected with the Corridorl and Corridorl connects the Elevator on Floorl.

5. The high level and low level symbols are connected by the entrance/exit symbols, like Elevator on Floor 1 in Figure 5.

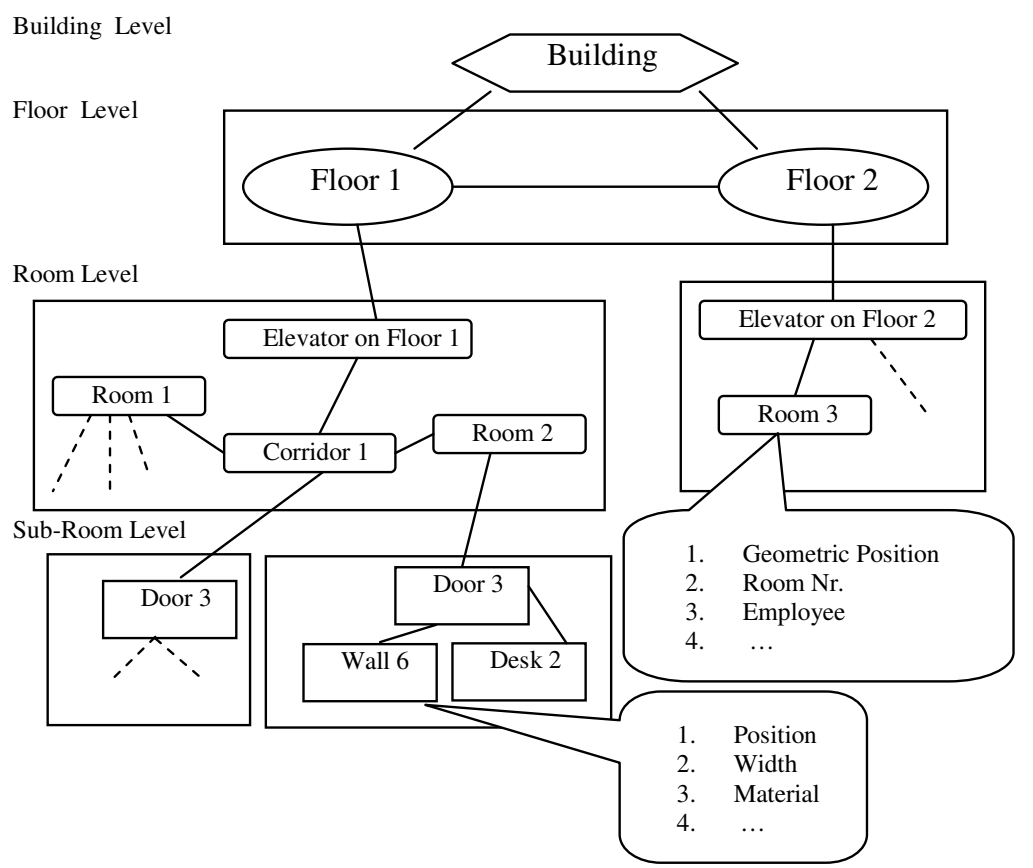

Fig. 5. Example of the Vector-Based Multi-Layer Map Structure.

Using this map representation, the wall or room information required by the localization and tracking algorithm can be easily extracted. They are modelled during the generation of the map structure. In addition, the semantic information can be fastly obtained. People can access the semantic information by simply checking the properties of the symbols. Also, routing algorithm can be efficiently implemented by the hybrid tree and graph structure. For example, if we want to walk from Room 2 to Room3, we can find the route: Room $2 \rightarrow$ Corridor $1 \rightarrow$ Elevator on Floor $1 \rightarrow$ Elevator on Floor $2 \rightarrow$ Room3. Last but not the least, this model also facilitates the information exchange with web service, i.e. the model can also be easily represented by XML or SVG format.

It is also not difficult to generate this model from another map format. For example, we can use the following procedures to generate the above mentioned map model from the raster JPEG or BMG images. 
Step 1: Identify the basic symbols, like walls or rooms. Since the symbols have a specific geometric shape, we can use the sophisticated image processing algorithms to identify the symbols. For complex building plans, manual efforts might also be needed.

Step 2: Incorporate the Semantic Information. For each symbol, the semantic information can be inputted as its property.

Step 3: Construct the symbol topology hierarchy. After recognizing the symbols, the topological structure can be conveniently extracted. For example, we can build the room level connection matrix by checking whether two rooms share the some wall or door.

Step 4: Store the structured location information in a database or XML file.

\section{Conclusions}

This paper comprehensively analyzes the role of map information in modern indoor localization systems. We present the basic principles of our previous work, which integrates map information into multiple layers of a WLAN localization system, from radio map generation, tracking, and fusion to semantic navigation. We also introduce a vector-based hybrid structured map mode, which represents the location information with the symbols layered according to their spatial relationship. We believe that this model can fulfill the requirements of the map-related algorithms, and hence maximize the contribution of the map information to the whole indoor location-aware systems.

\section{Acknowledgement}

The authors would like to thank Andreas Igel for his comments and suggestions to this paper.

\section{References}

1. C.A.Patterson, R.R. Muntz, and C.M. Pancake: Challenges in location-aware Computing. Pervasive Computing, vol.2, (2003) 80-89

2. Mark Weiser: Hot Topics: Ubiquitous Computing. IEEE Computer (1993)

3. Jeffrey Hightower and Gaetano Borriello: Location System for Ubiquitous Computing. IEEE computer (2001)

4. Hiroaki Koshima and Joseph Hoshen: Personal Locator Services Emerge. IEEE Spectrum (2000)

5. P. Bahl and V. N. Padmanabhan: RADAR: An in-building RF-based User Location and Tracking system. In proceedings of IEEE INFOCOM 2000, 2(3), pp. 775-784 (2000)

6. H. Wang: Fusion of Information Sources for Indoor Positioning with Field Strength Measurements. Master thesis, Department of Electrical Engineering and Information Technology, TU-München (2005) 
7. D. Obradovic, H. Lenz and M. Schupfner: Sensor Fusion in Siemens Car Navigation System. In proceedings of IEEE Conference on Machine Learning and Signal Processing, São Luis, Brazil(2004)

8. D. Obradovic, H. Lenz and M. Schupfner: Fusion of Map and Sensor Data in a Modern Car Navigation System. Journal of VLSI Signal Processing, Vol.45, p111-122 (2006)

9. G. Wölfle, R. Wahl, P. Wertz, P. Wildbolz, F. Landstorfer: Dominant Path Prediction Model for Indoor Scenarios. German Microwave Conference (GeMIC), Ulm, Germany, (2005)

10. Gergely V. Zarubo, Manfred Huber, Farhad A. Kamanger, and Imrich Chlamtac: Monte Carlo Sampling Based In-home Location Tracking With Minimal RF Infrastructure Requirements. In proceedings of GLOBECOM (2004)

11. H. Wang, H. Lenz, A. Szabo, J. Bamberger, U. D. Hanebeck: WLAN-Based Pedestrian Tracking Using Particle Filters and Low-Cost MEMS Sensors. In proceedings of 4th Workshop on Positioning, Navigation and Communication 2007 (WPNC'07), Hannover, Germany (2007)

12. Frédéric Evennou and Francois Marx: Advanced Integration of WiFi and Inertial Navigation Systems for Indoor Mobile Positioning. EURASIP Journal on Applied Signal Processing, Volume 2006, Article ID 86706, Pages 1-11(2006)

13. L. Liao, D. Fox, J. Hightower, H. Kautz, and D. Schulz: Voronoi Tracking: Location Estimation Using Sparse and Noisy Sensor Data. In Proceedings of IROS-03 (2003)

14. Frédéric Evennou, et al: Map-aided Indoor Mobile Positioning System using Particle Filter. In proceedings of $\mathrm{WCNC}(2005)$

15. H. Wang, H. Lenz, A. Szabo, U. D. Hanebeck, J. Bamberger: Fusion of Barometric Sensors, WLAN Signals and Building Information for 3-D Indoor/Campus Localization. In proceedings of International Conference on Multisensor Fusion and Integration for Intelligent Systems (MFI 2006), S. 426-432, Heidelberg, Germany (2006)

16. G. Retscher: Location Determination in Indoor Environments for Pedestrian Navigation", in proceedings of IEEE/ION PLANS 2006, San Diego, USA (2006)

17. Jürgen Bohn and Harald Vogt: Robust Probabilistic Positioning based on High-Level Sensor-Fusion and Map Knowledge. Technical Report, ETH, Zurich, Switzerland (2003)

18. http://usa.autodesk.com/adsk/servlet/index?siteID=123112\&id=6101753

19. S. Activity: Spatial Location BOF of IETF.http://www-nrc.nokia.com/ietf-spatial (2000)

20. U. Leonhardt: Supporting Location-awareness in Open Distributed Systems. PhD thesis, Department of Computing, Imperial College, London (1998)

21. A.K.Narayanan: Realms of States: a Framework for Location Aware Mobile Computing. In proceedings of the first international workshop on mobile commerce (2001)

22. Haibo Hu, Dik-Lun Lee: Semantic Location Modeling for Location Navigation in Mobile Environment. In proceedings of the 2004 IEEE international conference on mobile data management (MDM`04) (2004)

23. B.B. Parodi, H. Lenz, A. Szabo, H. Wang, J. Horn, J. Bamberger and J. Obradovic: Initialization and Online-Learning of RSS Maps for Indoor/Campus Localization. In proceedings of IEEE/ION PLANS 2006, San Diego, USA(2006)

24. B.B. Parodi, H. Lenz, A. Szabo, J. Bamberger and J. Horn: SLL: Statistical Conditions and Algebraic Properties. In proceedings of 4th Workshop on Positioning, Navigation and Communication 2007 (WPNC'07), Hannover, Germany (2007)

25. B.B. Parodi, H. Lenz, A. Szabo, J. Bamberger and J. Horn: Algebraic and Statistical Conditions for Use of SLL. In proceedings of European Control Conference 2007, Greece (2007)

26. Matthias Lott, Ingo Forkel: A Multi-Wall-and Floor Model for Indoor Radio Propagation. Vehicular Technology Conference (2001) 